\title{
PERIPHERALLY SPECIFIED HOMOMORPHS OF KNOT GROUPS
}

\author{
DENNIS JOHNSON AND CHARLES LIVINGSTON
}

\begin{abstract}
Let $G$ be a group and let $\mu$ and $\lambda$ be elements of $G$. Necessary and sufficient conditions are presented for the solution of the following problem: Is there a knot $K$ in $S^{3}$ and a representation $\rho: \pi_{1}\left(S^{3}-K\right) \rightarrow G$ such that $\rho(m)=\mu$ and $\rho(l)=\lambda$, where $m$ and $l$ are the meridian and longitude of $K$ ?
\end{abstract}

Let $G$ be a group and let $\mu$ and $\lambda$ be elements of $G$. Is there a knot $K$ in $S^{3}$ and a surjective homomorphism $\rho: \pi_{1}\left(S^{3}-K\right) \rightarrow G$ such that $\rho(m)=\mu$ and $\rho(l)=\lambda$, where $m$ and $l$ are the meridian and longitude of $K$ ? We will present necessary and sufficient conditions on the triple $(G, \mu, \lambda)$ for the positive solution of this problem.

An initial observation is that $G$ must be finitely generated and of weight one; $\mu$ must normally generate $G$. Fix $G$ and $\mu$ with these properties. If, for a given $\lambda \in G$, a knot $K$ and representation $\rho$ as above exist, we say that $\lambda$ is realizable. The set of realizable $\lambda$ forms a subgroup $R \subseteq G$.

Our main result is the following. The notation is described below.

Proposition 1. $\lambda \in R$ if and only if

(1) $\lambda \in G^{\prime \prime} \cap Z(\mu)$,

(2) $\langle\mu, \lambda\rangle=0 \in H_{2}(G)$,

(3) $\{\mu, \lambda\}=0 \in H_{3}\left(G / G^{\prime}\right) / p_{*}\left(H_{3}(G)\right)$.

In the above statement $G^{\prime \prime}$ denotes the second commutator subgroup of $G$ and $Z(\mu)$ is the centralizer of $\mu$. 〈, > denotes the Pontryagin product, which maps pairs of commuting elements of $G$ to $H_{2}(G) .\{$,$\} is a$ map which sends pairs of elements of $G$ satisfying conditions (1) and (2) to $H_{3}\left(G / G^{\prime}\right) / p_{*}\left(H_{3}(G)\right)$. $p: G \rightarrow G / G^{\prime}$ is the natural projection. For future reference, denote $H_{3}\left(G / G^{\prime}\right) / p_{*}\left(H_{3}(G)\right)$ by $Q$. All these notions will be defined in $\S 1$.

The necessity of these conditions will be rather straightforward to prove. The sufficiency will be implied by a stronger proposition. Let $P$ denote the set of $\lambda$ for which conditions (1) and (2) hold; it is a subgroup of $G$. Define $\chi: P \rightarrow Q$ by $\chi(\lambda)=\{\mu, \lambda\}$.

Received by the editors November 5, 1985.

1980 Mathematics Subject Classification (1985 Revision). Primary 57M25.

Supported in part by grants from the National Science Foundation. 
Proposition 2. $\chi$ is a surjective homomorphism with kernel $R$.

An interesting result that should be mentioned at this point is that for finite groups the condition of being weight one is equivalent to having cyclic abelianization. This is a result of Kutzko [8].

The history of this problem is as follows. Gonzalez-Acuna [4] proved that for a pair $(G, \mu)$ as above, there exist a knot $K$ in $S^{3}$ and a surjective representation $\rho: \pi_{1}\left(S^{3}-K\right) \rightarrow G$ such that $\rho(m)=\mu$. This result solved a problem posed by Neuwirth, question $U$ of [10]. Johnson [5] gave a second more direct proof of that result. His construction was discovered independently by Casson, in unpublished work. The necessity of conditions (1) and (2) in Proposition 1 was discovered by Johnson [6] in his extension of [5] and independently by Edmonds and Livingston [3] in their solution to a problem posed by Fox and Perko, question 1.24 of [7].

ACKNOWLEDGEMENTS. In the course of preparing [3] Edmonds saw the possibility of using a bordism argument. At the time we did not see how to apply such an approach. Johnson's work indicated the appropriate direction of attack. Conversations with Allan Edmonds have been extremely helpful in the development of this work. Thanks are due to Jim Van Buskirk and John Harer for their careful reading of Johnson's early work on this topic. Cameron Gordon produced the examples of groups for which $Q$ is nonzero. His examples are presented in Appendix 3, and we thank him for that contribution.

\section{Preliminaries}

For the most part, this work is independent of [3] and [6]. Some of the preliminary material described in this section is common to all three. We will outline the proofs of these basic results; the reader may fill in the details or consult the earlier works.

We will be working in the smooth, oriented category. A reference for knot theory and three-dimensional surgery is [11]. A reference for the bordism results is [2].

If $\gamma$ is a closed, based path in a space $X$, we will denote the class of that path in the fundamental group of the space by $\gamma$ as well.

In general, we will avoid references to basepoints for the fundamental groups of spaces. One comment is required. For a knot complement the basepoint can be chosen to lie on the boundary of a tubular neighborhood of the knot, the "peripheral torus." The meridian and longitude are defined in the usual way.

Let $K_{1}$ and $K_{2}$ be knots in $S^{3}$ with representations $\rho_{i}: \pi_{1}\left(S^{3}-K_{i}\right) \rightarrow G$ such that $\rho_{1}\left(m_{1}\right)=\rho_{2}\left(m_{2}\right)=\mu$ and $\rho_{i}\left(l_{i}\right)=\lambda_{i}$, where $m_{i}$ and $l_{i}$ are the meridian and longitude to $K_{i}$. There is a naturally defined homomorphism $\rho: \pi_{1}\left(S^{3}-K_{1} \# K_{2}\right) \rightarrow G$, with $\rho(m)=\mu$ and $\rho(l)=\lambda_{1} \lambda_{2}$, where $m$ and $l$ are the meridian and longitude to $K_{1} \# K_{2}$. This is the key to proving the next result. 
Theorem 1. The set of realizable $\lambda, R$, is a subgroup of $G$.

Proof. If $\lambda_{1}$ and $\lambda_{2}$ are realizable, then a connected sum can be used to show $\lambda_{1} \lambda_{2}$ is realizable. To realize $\lambda_{1}^{-1}$, use the mirror image of the knot used to realize $\lambda_{1}$, with orientation reversed. That $R$ is nonempty is the key result of $[4,5]$. It follows from a proof similar to the one we give for Theorem 3 as well.

Bordism results. Suppose that $\rho: \pi_{1}\left(M^{n}\right) \rightarrow G$ is a representation of the fundamental group of a closed, oriented $n$-manifold to $G$. There is an induced map of $H_{n}\left(M^{n}\right) \rightarrow H_{n}(G)$. Denote the image of the generator of $H_{n}\left(M^{n}\right)$ in $H_{n}(G)$ by $\left[M^{n}, \rho\right]$.

Recall that for $n=2$ or 3 the natural map $\Omega_{n}(G) \rightarrow H_{n}(G)$ is an isomorphism, where $\Omega_{n}(G)$ is the $G$-bordism group. Hence, if $n=2$ or 3 and $\left[M^{n}, \rho\right]=0 \in H_{n}(G)$, there exists a compact oriented $n+1$ manifold $W^{n+1}$ with a representation $\bar{\rho} ; \pi_{1}\left(W^{n+1}\right) \rightarrow G$ such that $\partial W^{n+1}=M^{n}$, and $\left.\bar{\rho}\right|_{\partial W^{n+1}}=\rho$.

Products. Given a commuting elements $g_{1}$ and $g_{2}$ of $G$ there is a natural homomorphism $\phi: Z \times Z \rightarrow G$ defined by $\phi((1,0))=g_{1}$ and $\phi((0,1))=g_{2}$. $\phi$ induces a representation $\rho: \pi_{1}\left(S^{1} \times S^{1}\right) \rightarrow G$. Set $\left\langle g_{1}, g_{2}\right\rangle=\left[S^{1} \times S^{1}, \rho\right] \in$ $H_{2}(G)$. This product is called the Pontryagin product. We will be using the facts that $\left\langle g_{1}, g_{2} g_{3}\right\rangle=\left\langle g_{1}, g_{2}\right\rangle+\left\langle g_{2}, g_{3}\right\rangle$, when defined, and that $\left\langle g_{1}, g_{1}\right\rangle=0$. Both these results are easily derived. A reference is [1].

The map of $G^{\prime \prime} \cap Z(\mu)$ to $H_{2}(G)$ defined by $\lambda \rightarrow\langle\mu, \lambda\rangle$ is a homomorphism. Denote the kernel by $P$.

Suppose now that $\lambda \in P$. Construct a 3-manifold $W^{3}$ with representation $\bar{\rho}: \pi_{1}\left(W^{3}\right) \rightarrow G$ such that $\partial W^{3}=S^{1} \times S^{1}$, and $\left.\bar{\rho}\right|_{\partial W^{3}}\left(\{\right.$ pt. $\left.\} \times S^{1}\right)=\mu$ and $\left.\bar{\rho}\right|_{\partial W^{3}}\left(S^{1} \times\{\right.$ pt. $\left.\}\right)=\lambda$ as described above.

Let $p: G \rightarrow G / G$ be the natural quotient map. The homomorphism $p \circ \bar{\rho}$ : $\pi_{1}\left(W^{3}\right) \rightarrow G / G^{\prime}$ extends to a representation $\psi: \pi_{1}\left(W^{3} \cup S^{1} \times B^{2}\right) \rightarrow G / G^{\prime}$, where $\partial W^{3}$ is identified with $\partial\left(S^{1} \times B^{2}\right)$ via a map that identifies $\{$ pt. $\} \times \partial B^{2} \subseteq$ $S^{1} \times B^{2}$ with $S^{1} \times\{\mathrm{pt}$. $\} \subseteq \partial W^{3}$. The extension exists by Van Kampen's Theorem, as attaching $S^{1} \times \bar{B}^{2}$ to $W$ in this way kills the longitude, which is the kernel of $p \circ \rho$. If we denote by $q$ the natural projection map $q: H_{3}\left(G / G^{\prime}\right) \rightarrow$ $Q=H_{3}\left(G / G^{\prime}\right) / p_{*}\left(H_{3}(G)\right)$, then define $\{\mu, \lambda\}=q\left(\left[W^{3} \cup S^{1} \times B^{2}, \psi\right]\right)$.

It will be left to the reader to check that $\{\mu, \lambda\}$ is well defined. Briefly, note that the choice of $W^{3}$ does not affect the value of $\{\mu, \lambda\}$, as we are working modulo $p_{*}\left(H_{3}(G)\right)$. A nontrivial observation is that $\left\{\mu, \lambda_{1} \lambda_{2}\right\}=\left\{\mu, \lambda_{1}\right\}+$ $\left\{\mu, \lambda_{2}\right\}$, when defined. The proof is contained in Appendix 1. It follows that $\chi: P \rightarrow Q$, defined by $\chi(\lambda)=\{\mu, \lambda\}$, is a homomorphism.

\section{Proposition 1, Necessity}

Suppose that we have a knot $K$ in $S^{3}$, with meridian $m$ and longitude $l$, and a representation $\rho: \pi_{1}\left(S^{3}-K\right) \rightarrow G$ such that $\rho(m)=\mu$ and $\rho(l)=\lambda$. 
We wish to verify that conditions (1), (2), and (3) of Proposition 1 hold for the pair $(\mu, \lambda)$.

Conditions (1) and (2) are straightforward. Observe that $l \in \pi_{1}\left(S^{3}-K\right)^{\prime \prime} \cap$ $Z(m)$. Also, $\langle m, l\rangle=0 \in H_{2}\left(\pi_{1}\left(S^{3}-K\right)\right)$, as $H_{2}\left(S^{3}-K\right)=0$, and hence $H_{2}\left(\pi_{1}\left(S^{3}-k\right)\right)=0$ as well. (Recall that $l \in \pi_{1}\left(S^{3}-K\right)^{\prime \prime}$ for the following reason: $l$ can be expressed as a commutator of elements of $\pi_{1}$ represented by curves on a Seifert surface for $K$. Any curve on a Seifert surface is itself in the commutator subgroup, as it lifts to the infinite cyclic cover of $K$. Also recall that $S^{3}-K$ is a $K(\pi, 1)$.)

The necessity of condition $(3),\{\mu, \lambda\}=0$, is slightly more difficult. $S^{3}-\stackrel{\circ}{N}$ $(K)$ can serve as the manifold $M^{3}$ used to define $\{\mu, \lambda\}$, where $\stackrel{\circ}{N}(K)$ is the interior of a regular neighborhood of $K . M^{3} \cup S^{1} \times B^{2}$ is 0-surgery on $K$. Denote it by $N^{3}$. We have a representation of $\pi_{1}\left(N^{3}\right) \rightarrow G / G$. This induces a map of $N^{3} \rightarrow K\left(G / G^{\prime}, 1\right) .\{\mu, \lambda\}$ is the image of the generator of $H_{3}\left(N^{3}\right)$ in $H_{3}\left(K\left(G / G^{\prime}, 1\right)\right)=H_{3}(G)$.

Since $H_{1}\left(N^{3}\right)=Z$ and $G / G$ is abelian, the above maps factor through maps to $Z$ and $K(Z, 1)$. The argument is completed by noting that $H_{3}(Z)=0$.

\section{3. $\chi$ IS SURJECTIVE}

In the case that $G^{\prime}=G$ or $G / G^{\prime}=Z$ it holds that $Q$ is trivial. Hence, assume that $G / G^{\prime}=Z_{n}$, with $n>1$. In this case $H_{3}\left(G / G^{\prime}\right)=Z_{n}$ as well.

In order to show that $\chi$ is surjective we need the fact that $\mu^{n} \in P$. The only difficult observation here is that $\mu^{n} \in G^{\prime \prime}$; it is only clear that $\mu^{n} \in G^{\prime}$. We leave the proof of this purely algebraic theorem to Appendix 2 .

Given this, we claim that $\chi\left(\mu^{n}\right)$ is a generator of $Q$. The map $\phi$ : $\pi_{1}\left(S^{1} \times S^{1}\right) \rightarrow G$ defined by $\phi\left(\{\right.$ pt. $\left.\} \times S^{1}\right)=\mu$ and $\phi\left(S^{1} \times\{\right.$ pt. $\left.\}\right)=\mu^{n}$ vanishes on a nontrivial simple closed curve on $S^{1} \times S^{1}$. Hence, it is easy to see that $\phi$ extends to a map of $\pi_{1}\left(S^{1} \times B^{2}\right)$ to $G$. (Warning: the identification of $S^{1} \times S^{1}$ with $\partial\left(S^{1} \times B^{2}\right)$ is not the standard one!) (This proves $\left\langle\mu, \mu^{n}\right\rangle=0$ also.)

It follows that the manifold $M^{3}$ used in defining $\left\{\mu, \mu^{n}\right\}$ can be taken to be $S^{1} \times B^{2}$. Hence the manifold $M^{3} \cup S^{1} \times B^{2}$ is a lens space. If the construction is done carefully one sees that $M^{2} \cup S^{1} \times B^{2}$ can be taken to be the lens space $L(n, 1)$.

The final result needed is that if $\psi: \pi_{1}(L(n, 1)) \rightarrow Z_{n}$ is surjective then the image of the generator of $\mathrm{H}_{3}(L(n, 1))$ in $\mathrm{H}_{3}\left(Z_{n}\right)$ under the induced map of $L(n, 1)$ to $K\left(Z_{n}, 1\right)$ is a generator. Recalling that $L(n, 1)$ is a threedimensional approximation to $K\left(Z_{n}, 1\right)$, the result is immediate.

Corollary. $\chi\left(\mu^{h n}\right)$ is the reduction of $h$ times a generator of $H_{3}(G / G)$ in $Q$. 


\section{Preliminary Realization Results}

At this point it remains to show that if $\lambda \in \operatorname{Ker}(\chi)$ then $\lambda \in R$. In this section we will prove two preliminary realization results.

Theorem 2. $\mu^{n^{2}} \in R$, where $n$ is the order of $G / G^{\prime}$.

Proof. Let $K$ be a knot in $S^{3}$ with meridional curve $m$ and longitude $l$. Let $C$ be an unknotted curve in $S^{3}-K$. If +1 surgery is performed on $S^{3}$ along $C$ the resulting 3-manifold is again $S^{3}$. K represents a new knot $K^{\prime}$ in the manifold $S^{3}$ after the surgery. The curve $m$ represents a meridian to $K^{\prime}$. However, $l$ may not be a longitude to $K^{\prime}$. A simple calculation shows that the longitude of $K^{\prime}, l^{\prime}$, is homotopic to $l \cdot m^{-d^{2}}$ in $S^{3}-K^{\prime}$, where $d$ is the linking number of $K$ and $C$.

Now suppose that $K$ is a knot in $S^{3}$ such that there is a representation $\rho$ : $\pi_{1}\left(S^{3}-K\right) \rightarrow G$ with $\rho(m)=\mu$ and $\rho(l)=1$ as provided by Theorem 1 . In order to prove Theorem 2 we must produce a curve $C$ as above, with $\rho(C)=1$ and with the linking number of $C$ with $K$ being $n$. With $C$ satisfying these properties there will be representation $\rho^{\prime}: \pi_{1}\left(S^{3}-K^{\prime}\right)-G$, with $\rho^{\prime}\left(l^{\prime}\right)=\mu^{-\left(n^{2}\right)}$, which suffices for the proof of Theorem 2 .

Arranging $C$ to be unknotted is not difficult. Observe that if $J_{1} \cup J_{2}$ is any link in $S^{3}, J_{2}$ is homotopic in $S^{3}-J_{1}$ to a curve which is unknotted in $S^{3}$.

It is easy to find a simple closed curve $C_{1}$ in $S^{3}-K$ with $\rho\left(C_{1}\right)=\mu^{n}$ and with linking number $n$ with $K$. Just take $m^{n}$ and perform a homotopy to make it embedded. If we could find a second simple closed curve $C_{2}$ with $\rho\left(C_{2}\right)=\mu^{n}$ and with linking number 0 with $K$ we would be done. Just set $C$ to be the connected sum of $C_{1}$ with an orientation reversed $C_{2}$. We need the following algebraic result.

Claim. If $g \in G^{\prime}$, then $g=\Pi_{i} h_{i} \mu^{\varepsilon_{i}} h_{i}^{-1}$ with $h_{i} \in G$ and $\sum_{i} \varepsilon_{i}=0$.

Proof of Claim. One need only check this for a commutator $\left[g_{1}, g_{2}\right]$. The result follows immediately on writing $g_{1}$ and $g_{2}$ as products of conjugates of $\mu$.

To complete the proof of Theorem 2, note that $\mu^{n} \in G^{\prime}$. Hence $\mu^{n}=$ $\Pi_{i} h_{i} \mu_{i}^{\varepsilon_{i}} h_{i}^{-1}$, with $\sum_{i} \varepsilon_{i}=0$. Pick for convenience a basepoint for $\pi_{1}\left(S^{3}-K\right)$ on $m$. Pick closed paths $\gamma_{i}$ with $\rho\left(\gamma_{i}\right)=h_{i}$. Set $\gamma=\Pi_{i} \gamma_{i} m^{\varepsilon_{i}} \gamma_{i}^{-1} \cdot \rho(\gamma)=\mu^{n}$ as desired. In addition, it is clear that $\gamma$ links $K$ algebraically 0 times. Perform a homotopy to $\gamma$ to make it embedded. This yields the desired curve $C_{2}$, completing the proof of Theorem 2 .

Theorem 3. If $\lambda \in P$ then $\lambda \mu^{h n} \in R$, for some $h \in Z$.

Proof. As $\langle\mu, \lambda\rangle=0$, there are a compact oriented 3-manifold $M^{3}$, with $\partial M^{3} \cong S^{1} \times S^{1}$, and a representation $\rho: \pi_{1}\left(M^{3}\right) \rightarrow G$ such that $\rho\left(\{\right.$ pt. $\left.\} \times S^{1}\right)=$ $\mu$ and $\rho\left(S^{1} \times\{\right.$ pt. $\left.\}\right)=\lambda$. Denote $\{$ pt. $\} \times S^{1}$ by $m$ and $S^{1} \times\{$ pt. $\}$ by $l$. By 
forming the connected sum of $M^{3}$ with copies of $S^{1} \times S^{2}$ we add free generators to $\pi_{1}\left(M^{3}\right)$ and hence can arrange that $\rho$ is surjective.

Form the closed 3-manifold $W^{3}$ as $M^{3} \cup S^{1} \times B^{2}$, with the natural identification. $M^{3}$ is the complement of the knot $K=S^{1} \times\{0\} \subseteq S^{1} \times B^{2} \subseteq W^{3}$. The meridian to $K$ is $m$.

We now wish to do surgery on $W^{3}$, in the complement of $K$, to reduce it to $S^{3}$. This is certainly possible, as every closed oriented 3-manifold is obtained by surgery on a link in $S^{3}[9,12]$. The delicate point is to do the surgery in such a way that we can maintain a representation to $G$ throughout and not change its behavior on the peripheral subgroup. This will be possible if we perform surgery only on curves $\gamma_{i}$ for which $\rho\left(\gamma_{i}\right)=1$. The following indicates that this is possible.

Claim. Any simple closed curve $\gamma \subseteq M^{3}$ is isotopic, in $W^{3}$, to a curve $\gamma^{\prime} \subseteq M^{3}$ such that $\rho\left(\gamma^{\prime}\right)=1$.
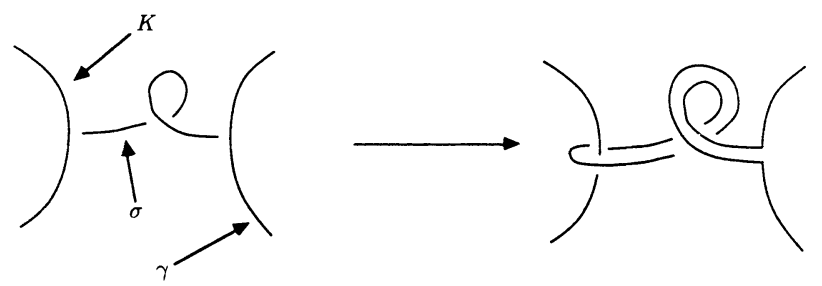

FIGURE 1

Proof of Claim. Pick a path $\sigma$ from $\gamma$ to $K$. Perform an isotopy of $\gamma$ along $\sigma$ to $K$ and then across $K$, as illustrated in Figure 1. The effect is to multiply the element of $\pi_{1}\left(M^{3}\right)$ represented by $\gamma$ by a conjugate of $m$. The choice of $\sigma$ determines which conjugate. $\rho(\gamma)$ is multiplied by a conjugate of $\mu$, determined by " $\rho(\sigma)$." (Basepoints must be selected in order for $\sigma$ to represent an element of $\pi_{1}\left(M^{3}\right)$.) As $\rho$ is surjective, we can multiply by any conjugate of $\mu . \rho(\gamma)$ can be written as a finite product of conjugates of $\mu$, so repeating the above procedure yields the desired result.

The preceding claim indicates that it is possible to perform the required surgery over $G$. We now have a knot $K \subseteq S^{3}$ and a surjective representation $\rho: \pi_{1}\left(S^{3}-K\right) \rightarrow G$. In addition, there are curves $m$ and $l$ on the peripheral torus to $K$ wth $\rho(m)=\mu$ and $\rho(l)=\lambda$. Now $m$ represents a meridian of $K$, but $l$ may not be a longitude of $K$. If we denote the meridian and longitude of $K$ by $m^{\prime}$ and $l^{\prime}$, we have $m^{\prime}=m$ and $l^{\prime}=l \cdot m^{k}$, for some integer $k$, as elements of $\pi_{1}\left(S^{3}-K\right)$. Hence $\rho\left(m^{\prime}\right)=\mu$ and $\rho\left(l^{\prime}\right)=\lambda \mu^{k}$. This implies that $\lambda \mu^{k} \in G^{\prime}$. Since $\lambda \in G^{\prime}$ it follows that $\mu^{k} \in G^{\prime}$ and that $k$ is divisible by $n$. This completes the proof of Theorem 3 . 


\section{Proof of Proposition 2}

Before proving Proposition 2 we need one technical lemma.

Lemma. Let $W^{3}$ be a closed, oriented 3-manifold with $H_{1}\left(W^{3}\right)=Z_{k \cdot n}$, and let $p: \pi_{1}\left(W^{3}\right) \rightarrow Z_{n}$ be the natural homomorphism. Then $\left[W^{3}, p\right] \in H_{3}\left(Z_{n}\right)$ is $k$ times a generator of $H_{3}\left(Z_{n}\right)$.

Proof. Since $p$ factors through a map to $Z_{k n}$, we can verify this lemma in two steps. First we show that the lemma holds if $k=1$. We conclude the argument by noting that the map $H_{3}\left(Z_{k \cdot n}\right) \rightarrow H_{3}\left(Z_{n}\right)$ induced by the quotient map sends a generator of $H_{3}\left(Z_{k \cdot n}\right)$ to $k$ times a generator of $H_{3}\left(Z_{n}\right)$. This follows from basic techniques of homological algebra [1].

Assume $k=1$. To show $p_{*}$ maps a generator of $H_{3}\left(W^{3}\right)$ to a generator of $H_{3}\left(Z_{n}\right)$, it is enough to check this with $Z_{n}$ coefficients. Working dually, we show that $p^{*}: H^{3}\left(Z_{n}, Z_{n}\right) \rightarrow H^{3}\left(W^{3}, Z_{n}\right)$ is an isomorphism. If we let $\beta$ denote the Bockstein, then $H^{3}\left(W^{3}, Z_{n}\right)$ is generated by $x \cup \beta(x)$, where $x$ is any generator of $H^{1}\left(W^{3}, Z_{n}\right)$. This follows from Poincare duality. Similarly, $H^{3}\left(Z_{n}, Z_{n}\right)$ is generated by $y \cup \beta(y)$, where $y$ is any generator of $H^{1}\left(Z_{n}, Z_{n}\right)$. This is well known and follows from Poincare duality for lens spaces. The result now follows from the observation that $p^{*}(y \cup \beta(y))=p^{*} y \cup \beta\left(p^{*} y\right)$, and if $y$ generates $H^{1}\left(Z_{n}, Z_{n}\right)$, then $p^{*}(y)$ generates $H^{1}\left(W^{3}, Z_{n}\right)$.

To prove Proposition 2 we show directly that if $\lambda \in P$ and $\chi(\lambda)=0$, then $\lambda \in R$.

Observe that by Theorem 3, if $\lambda \in P$ then $\lambda \mu^{h n} \in R$ for some $h$. Hence $\chi\left(\mu^{h n}\right)=0$. The proof is now reduced to the following.

Theorem 4. If $\chi\left(\mu^{h n}\right)=0$, then $\mu^{h n} \in R$.

Proof. Recall that $\left\{\mu, \mu^{h n}\right\}$ represents the reduction of $h$ times a generator of $H_{3}\left(G / G^{\prime}\right)$ in $Q$. Since $\left\{\mu, \mu^{h n}\right\}=0 \in Q$, there are a closed, oriented 3manifold $M^{3}$ and a representation $\rho: \pi_{1}\left(M^{3}\right) \rightarrow G$ such that $p_{*}\left(\left[M^{3}, \rho\right]\right)$ is $h$ times a generator of $H_{3}\left(G / G^{\prime}\right)$. By forming the connected sum with $S^{1} \times S^{2}$ we can arrange that $\rho$ is surjective. Since any representation of $\pi_{1}\left(S^{2} \times S^{2}\right)$ to $G$ extends to one of $\pi_{1}\left(S^{1} \times B^{3}\right)$ to $G$, this will not alter the class $\left[M^{3}, \rho\right]$.

Let $K$ be a trivial, unknotted circle in $M^{3}$. Denote the meridian and logitude of $K$ by $m$ and $l$. Extend $\rho$ to a representation $\rho^{\prime}: \pi_{1}\left(M^{3}-K\right) \rightarrow G$ by setting $\rho^{\prime}(m)=\mu$. Of course, $\rho^{\prime}(l)=1$.

Perform surgery on $M^{3}-K$ over $G$ to reduce it to a knot complement in $S^{3}$, as in the proof of Theorem 3. Call that knot, in $S^{3}, J$. Denote the images of $m$ and $l$ in $S^{3}-J$ by $m$ and $l$ as well. We have a representation $\sigma: \pi_{1}\left(S^{3}-J\right) \rightarrow G$ such that $\sigma(m)=\mu$ and $\sigma(l)=1$. 
Observe that if $m_{2}$ and $l_{2}$ denote the meridian and longitude to $J, m=m_{2}$ and $l=l_{2} \cdot m^{k \cdot n}$ for some $k$. (The power of $m$ must be divisible by $n$ since $\sigma(l)$ and $\sigma\left(l_{2}\right)$ are both in $G^{\prime}$.) To analyze $k$, proceed as follows.

Consider the closed manifold $N_{1}=\left(M^{3}-\stackrel{\circ}{N}(k)\right) \cup S^{1} \times B^{2}$ where the meridian to $S^{1} \times B^{2}$ is identified to $l . \rho^{\prime}$ induces a representation $\rho_{1}: \pi_{1}\left(N_{1}\right) \rightarrow G$. As $N_{1}=M^{3} \# S^{1} \times S^{2},\left[N_{1}, \rho_{1}\right]=\left[M^{3}, \rho\right] \in H_{3}(G)$.

Similarly, define the closed manifold $\stackrel{\circ}{N}_{2}=\left(S^{3}-N(J)\right) \cup S^{1} \times B^{2}$, identifying a meridian of $S^{1} \times B^{2}$ to $l$. $\sigma$ induces a representation $\sigma_{1}: \pi_{1}\left(N_{2}\right) \rightarrow G$. Observe that $\left[N_{2}, \sigma_{1}\right]=\left[N_{1}, \rho_{1}\right]$, as the classes are bordant over $G$.

In particular, projecting to $G / G$, we see that $p_{*}\left(\left[N_{2}, \sigma_{1}\right]\right) \in H_{3}\left(G / G^{\prime}\right)$ represents $h$ times a generator. On the other hand, $H_{1}\left(N_{2}\right)=Z_{k \cdot n}$, so the lemma applies. Hence, $p_{*}\left(\left[N_{2}, \sigma_{1}\right]\right)$ represents $k$ times some, perhaps different, generator of $H_{3}\left(G / G^{\prime}\right)$.

Denoting the two generators by $g_{1}$ and $g_{2}$, we have that $h \cdot g_{1}=k \cdot g_{2}$ in $Z_{n}$. Since $g_{1}$ is invertible in $Z_{n}$, we have $h=k \cdot g_{0}$ in $Z_{n}$ for some $g_{0} \in Z$. It follows that $h=k \cdot a+b \cdot n$ for some $a$ and $b$. Since $\mu^{k n} \in R$ (use the knot $J$ and representation $\sigma$ to show $\mu^{-k n} \in R$ ) and $\mu^{\left(n^{2}\right)} \in R$ (by Theorem 2), it follows from Theorem 1 that $\mu^{a k n+b\left(n^{2}\right)}=\mu^{h n}$ is in $R$ as well. This completes the proof of Proposition 2.

\section{Corollaries}

As an application of the above results we describe situations in which $P=$ $R$. Notice that by Theorem 3 , showing that $P=R$ reduces to showing that $\mu^{n} \in R$. The results of this section were originally proved in [6].

Corollary 1. If $G=G^{\prime}$, then $P=R$.

Proof. One proof is to note that $Q=0$. A second is to observe that $\mu \in R$, by Theorem 2.

Corollary 2. If $G / G^{\prime} \cong Z$, then $P=R$.

Proof. Again, $Q=0$. As a second approach, Theorem 3 can be applied directly.

Although $P$ and $R$ are defined in terms of $\mu$, note that $P / R$ is independent of $\mu$, being isomorphic to $Q$.

Corollary 3. If $G$ is normally generated by an element of order $n$, then $Q=0$. Proof. Let $\mu$ be that normal generator. Then $\mu^{n} \in R$, so $P=R$. Hence $P / R \cong Q=0$.

An application of Corollary 3 is to the group $S_{m}$.

We will discuss in Appendix 3 examples by Gordon of groups $G$ which are finitely generated and of weight one for which $Q \neq 0$. We should note here that there are finitely generated groups of weight one for which $Q=0$, but Corollary 3 does not apply. Johnson discovered the following interesting one 
in [6]. Let $G=\mathrm{SL}_{2}(Z) . G / G \cong Z_{12}$, but $G$ does not contain an element of order 12. The proof that for $\mathrm{SL}_{2}(Z), Q=0$ is contained in [6].

\section{AppendiX 1. AdDitivity of $\{$,}

It is our goal here to show that if $\lambda_{1}$ and $\lambda_{2}$ are in $P$, then $\left\{\mu, \lambda_{1} \lambda_{2}\right\}=$ $\left\{\mu, \lambda_{1}\right\}+\left\{\mu, \lambda_{2}\right\}$. We will prove that $\left\{\mu, \lambda_{1} \lambda_{2}\right\}-\left\{\mu, \lambda_{1}\right\}-\left\{\mu, \lambda_{2}\right\}$ is represented by a representation of $\pi_{1}\left(S^{1} \times S^{2}\right)$ to $G / G^{\prime}$. It is hence trivial in $Q$, as any representation of $\pi_{1}\left(S^{1} \times S^{2}\right)$ extends to one of $\pi_{1}\left(S^{1} \times B^{3}\right)$.

We will view representations of the fundamental group of spaces as corresponding to maps of those spaces into Eilenberg-Mac Lane spaces. Details concerning choice of basepoints will be left to the reader. The manifold used to compute $\left\{\mu, \lambda_{1} \lambda_{2}\right\}$ will be constructed from those used to compute $\left\{\mu, \lambda_{1}\right\}$ and $\left\{\mu, \lambda_{2}\right\}$. In the following, $p: K(G, 1) \rightarrow K(G / G, 1)$ is the natural projection. The schematic illustration, Figure 2, may be of assistance in following the argument.

There exist 3-manifolds $M_{1}, M_{2}$ with maps $f_{i}: M_{i} \rightarrow K(G, 1)$ such that $\partial M_{i} \cong S^{1} \times S^{1}$ and $f_{i^{*}}\left(\{\right.$ pt. $\left.\} \times S^{1}\right)=\mu \in \pi_{1}(K(G, 1))=G$ and $f_{i^{*}}\left(S^{1} \times\{\right.$ pt. $\left.\}\right)$ $=\lambda_{i} \in G . \quad\left(\{\mathrm{pt}.\} \times S^{1}\right.$ and $S^{1} \times\{$ pt. $\}$ are homotopy classes of paths represented by curves in $\partial M_{i}$.) Denote by $N_{i}$ the 3-manifold $M_{i} \cup S^{1} \times B^{2}$, with the boundaries being identified by maps sending the meridian of $S^{1} \times B^{2}$ to $S^{1} \times$ \{pt. $\subseteq \partial M_{i}$ and the longitude of $S^{1} \times B^{2}$ to $\{$ pt. $\} \times S^{1} \subseteq \partial M_{i}$. There are naturally defined maps of $N_{i}$ to $K(G / G, 1)$, which we denote by $\bar{f}_{i}$.

Construct $M_{3}$ as follows. Let $A_{i}$ be an annular neighborhood of $\{$ pt. $\} \times S^{1} \subseteq$ $\partial M_{i}$. Let $M_{3}$ be the 3-manifold $M_{1} \cup A \times I \cup M_{2}$, where $A \times\{0\}$ is identified with $A_{1} \subseteq \partial M_{1}$ and $A \times\{1\}$ is identified with $A_{2} \subseteq \partial M_{2}$. The maps $f_{1}$ and $f_{2}$ extend to a map $f_{3}$ of $M_{3}$ to $K(G, 1)$. Note that $\partial M_{3} \cong S^{1} \times S^{1}$ and, with the natural identification of $\partial M_{3}$ with $S^{1} \times S^{1}, f_{3^{*}}\left(\{\right.$ pt. $\left.\} \times S^{1}\right)=\mu$ and $f_{3^{*}}\left(S^{1} \times\{\right.$ pt. $\left.\}\right)=\lambda_{1} \lambda_{2}$.

Denote by $N_{3}$ the manifold $M_{3} \cup S^{1} \times B^{2}$, with boundaries identified as in the construction of $N_{1}$ and $N_{2}$. Let $\bar{f}_{3}$ denote the natural map of $N_{3}$ to $K\left(G / G^{\prime}, 1\right)$. We will show that the class of $H_{3}(G / G),\left[N_{3}, \bar{f}_{3}\right]-\left[N_{1}, \bar{f}_{1}\right]-$ $\left[N_{2}, \bar{f}_{2}\right]$, is represented by a map of $S^{1} \times S^{2}$ to $K\left(G / G^{\prime}, 1\right)$.

We can view $M_{1}$ and $M_{2}$ as being contained in $N_{3}$ as well as in $N_{1}$ and $N_{2}$, respectively. Further, $\bar{f}_{3 \mid M_{i}}=\bar{f}_{i \mid M_{i}}$.

$N_{3}-M_{1}-M_{2} \cong S^{1} \times B^{2} \cup A \times I$, with proper boundary identifications. Denote the $S^{1} \times B^{2}$ by $\left(S^{1} \times B^{2}\right)_{3}$. Similarly, $N_{i}-M_{i} \cong S^{1} \times B^{2}$. Denote these copies of $S^{1} \times B^{2}$ by $\left(S^{1} \times B^{2}\right)_{i}$.

We have the equality $\left[N_{3}, \bar{f}_{3}\right]-\left[N_{1}, \bar{f}_{1}\right]-\left[N_{2}, \bar{f}_{2}\right]=\left[M_{1} \cup M_{2} \cup A \times I \cup\right.$ $\left.\left(S^{1} \times B^{2}\right)_{3}, \bar{f}_{3}\right]-\left[M_{1} \cup\left(S^{1} \times B^{2}\right)_{1}, \bar{f}_{1}\right]-\left[M_{2} \cup\left(S^{1} \times B^{2}\right)_{2}, \bar{f}_{2}\right]$. Cancellation 


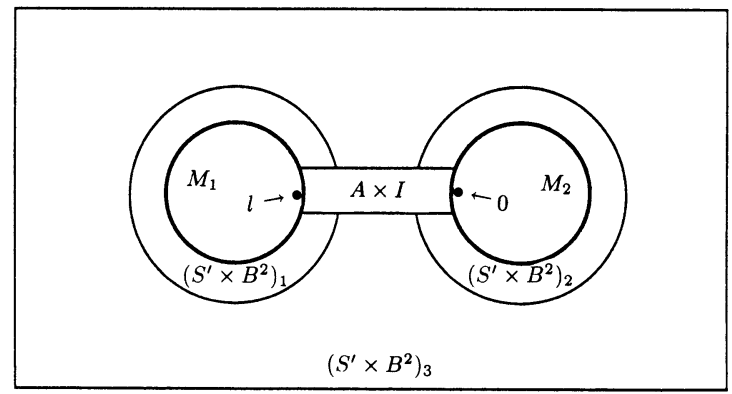

FIGURE 2

can occur in the difference, and we see that the class being studied is represented by the appropriate map, involving restrictions of the $\bar{f}_{i}$, of $\left(S^{1} \times B^{2}\right)_{1} \cup$ $\left(S^{1} \times B^{2}\right)_{2} \cup\left(S^{1} \times B^{2}\right)_{3} \cup A \times I$ to $K\left(G / G^{\prime}, 1\right)$, as now described.

The map $\bar{f}_{i}, i=1$ or 2 , and $\bar{f}_{3}$ agree on longitudinal annuli of $\left(S^{1} \times B^{2}\right)_{i}$ and $\left(S^{1} \times B^{2}\right)_{3}$. Hence $\bar{f}_{1}, \bar{f}_{2}$, and $\bar{f}_{3}$ together define a map of $\left(S^{1} \times B^{2}\right)_{1} \cup$ $\left(S^{1} \times B^{2}\right)_{2} \cup\left(S^{1} \times B^{2}\right)_{3}$ to $K\left(G / G^{\prime}, 1\right)$ where the union is along boundary annuli. The resulting space is diffeomorphic to $S^{1} \times B^{2}$. Hence, the class we are studying is represented by a map of $S^{1} \times B^{2} \cup A \times I$ to $K\left(G / G^{\prime}, 1\right)$. Note that $A \times I \cong S^{1} \times B^{2}$. The final identification of boundaries maps longitudes to longitudes and hence yields $S^{1} \times S^{2}$, as desired.

\section{APPENDIX 2. $G^{\prime \prime} \cap Z(\mu)=G \cap Z(\mu)$}

The following argument is taken directly from [6].

We wish to prove that for a finitely generated group $G$ of weight one, $G^{\prime \prime} \cap$ $Z(\mu)=G^{\prime} \cap Z(\mu)$, where $\mu$ is a normal generator of $G$. In other words, if $[\mu, x]=1$ and $x \in G^{\prime}$ then $x \in G^{\prime \prime}$. We shall actually prove a stronger statement: $[\mu, x] \in G^{\prime \prime}$ and $x \in G^{\prime} \Rightarrow x \in G^{\prime \prime}$. The latter is equivalent to $[\mu, x]=1$ in $G / G^{\prime \prime}$ and $x \in G^{\prime} / G^{\prime \prime} \Rightarrow x=1$ in $G / G^{\prime \prime}$. Thus it suffices to show that if $G^{\prime \prime}=1$ and $G$ is finitely generated by the conjugates of $\mu$, then $[\mu, x]=1$ and $x \in G^{\prime} \Rightarrow x=1$.

Proof. Since $G^{\prime \prime}=1, G^{\prime}$ is abelian and (if we write it additively) is therefore a $G / G^{\prime}$ module. The latter group is cyclic with generator $t$ which is the image of $\mu$; we write the ring $Z\left(G / G^{\prime}\right)$ simply as $Z[t]$. Note that, by definition, $(t-1) x=[\mu, x]$ for $x \in G$, where on the left we are using the additive notation and module multiplication and on the right the multiplicative notation in $G$.

Next we note that the group $(t-1) G^{\prime} \subset G^{\prime}$ is normal in $G$ (it is in fact $\left.\left[G, G^{\prime}\right]\right)$ and $G /(t-1) G^{\prime}$ is abelian, since in this quotient $H, \mu$ commutes 
with all of $H^{\prime}$ and also with the powers of $\mu$, which together generate $H$. Thus $(t-1) G^{\prime} \supset G^{\prime}$ and hence $(t-1) G^{\prime}=G^{\prime}$. Hence multiplication by $t-1$ in $G^{\prime}$ is a surjective module endomorphism. The statement we seek to prove may be rephrased in additive notation as $x \in G$ and $(t-1) x=0 \Rightarrow x=0$, so we are trying to show that multiplication by $t-1$ is also $1-1$. To this end we prove

Lemma. Let $M$ be a finitely generated module over a Noetherian ring and let $f$ be a surjective endomorphism of $M$. Then $f$ is an isomorphism.

Proof. Let $K_{1}=\operatorname{Ker} f$ and suppose $K_{1} \neq 0$. Put $K_{n}=\operatorname{Ker} f^{n}=\left(f^{n-1}\right)^{-1} K_{1}$. Since $f^{n-1}$ is surjective, $\left(f^{n-1}\right)^{-1} K_{1} \supsetneq\left(f^{n-1}\right)^{-1}(0)=K_{n-1}$. Thus we get $K_{1} \varsubsetneqq K_{2} \varsubsetneqq K_{3} \varsubsetneqq \cdots$. This is impossible since a finitely generated module over a Noetherian ring satisfies the ascending chain condition.

Since $Z[t]$ is Noetherian, the lemma gives us the desired proof if we can show that $G^{\prime}$ is finitely generated as a module over $Z[t]$ (it is not necessarily a finitely generated abelian group). By hypothesis, $G$ is generated by a finite number of elements of the form $\mu x_{1}, \mu x_{2}, \ldots, \mu x_{n}$, where $x_{i} \in G^{\prime}$. If $x \in G^{\prime}$, write it as a word in the above elements. Commuting the $\mu$ 's across the $x_{i}$ 's gives a word of the form $\mu^{n}$. (word in $t^{n_{i}} x_{i}$ 's). If $\mu^{m}$ is the smallest power of $\mu$ in $G^{\prime}$, then $x$ is clearly in the submodule of $G^{\prime}$ generated by $\mu^{m}$ and the $x_{i}$ 's. This shows that $G^{\prime}$ is finitely generated as a module over $Z[t]$, as required.

\section{APPENDix 3. EXAMPLes of $Q \neq 0$}

This appendix presents examples produced by Cameron Gordon.

$G$ will be a free product with amalgamation, $F_{1} *_{F_{3}} F_{2}$, with $F_{1}$ and $F_{2}$ (and hence $F_{3}$ ) free. Since $H_{i}(F)=0, i \geq 2$, for any free group $F$, any such $G$ will have $H_{i}(G)=0, i \geq 3$, by a Mayer-Vietoris argument. For the examples below, $H_{2}(G)=0$ as well. Fix an $n \geq 1$. Let $G$ have the following presentation:

$$
\begin{aligned}
G=\left\langle a, b, x, y: b^{-1} a^{n} b=[x, y], b^{-2} a^{n} b^{2}\right. & =y^{-2} x y^{2}, \\
a^{-1} b a & \left.=y^{-3} x y^{3}, a^{-2} b a^{2}=x^{-2} y x^{2}\right\rangle .
\end{aligned}
$$

We have immediately that $G$ is finitely generated and that $G=\langle a\rangle$. Also $G / G^{\prime}=Z_{n}$.

Let $F_{1}$ be the free group on $\{a, b\}, F_{2}$ be the free group on $\{x, y\}$, and $F_{3}$ be the free group of rank 4 on the elements given in the four equalities. One has to check that the elements on the left (right) are a basis for a free subgroup of $F_{1}\left(F_{2}\right)$. This is clear: call the elements on the left $A, B, C$, and $D$, and consider a reduced word in $A, B, C$, and $D$. Such a word obviously has nonzero length as a word in $a$ and $b$. (Just look at the maximal consolidations that can occur.) Similarly for the elements on the right. 


\section{REFERENCES}

1. K. Brown, Cohomology of groups, Springer-Verlag, New York, Heidelberg and Berlin, 1982.

2. P. Conner and E. Floyd, Differentiable periodic maps, Springer-Verlag, New York, 1964.

3. A. Edmonds and C. Livingston, Symmetric representations of knot groups, Topology Appl. 18 (1984), 281-314.

4. F. Gonzalez-Acuna, Homomorphs of knot groups, Ann. of Math. (2) 102 (1975), 373-377.

5. D. Johnson, Homomorphs of knot groups, Proc. Amer. Math. Soc. 78 (1980), 135-138.

6. __ Peripherally specified homomorphs of knot groups, preprint.

7. R. Kirby, Problems in low-dimensional manifold theory, Proc. Sympos. Pure Math., vol. 32, Amer. Math. Soc., Providence, R. I., 1978, pp. 273-312.

8. P. Kutzko, On groups of finite weight, Proc. Amer. Math. Soc. 55 (1976), 279-280.

9. W. B. R. Lickorish, A representation of orientable combinatorial 3-manifolds, Ann. of Math. 76 (1962), 531-538.

10. L. P. Neuwirth, Knot groups, Princeton Univ. Press, 1965.

11. D. Rolfsen, Knots and links, Publish or Perish, Berkeley, Calif., 1976.

12. A. D. Wallace, Modifications and cobounding manifolds, Canad. J. Math 12 (1960), 503-528.

Department of Mathematics, Indiana University, Bloomington, Indiana 47405 (Charles Livingston) 